\title{
Ambulatory management of primary spontaneous pneumothorax: the good is (not always) the enemy of the better levidence)
}

\author{
Arschang Valipour
}

Affiliation: Otto-Wagner-Spital, Vienna, Austria.

Correspondence: A. Valipour, Otto-Wagner-Spital, Sanatoriumsstrasse 2, 1140 Wien, Austria. E-mail: arschang.valipour@wienkav.at

0 @ERSpublications

We need to do a better job in the future to achieve the best care for our patients with primary spontaneous pneumothorax http://ow.ly/q1Y29

Pneumothorax is defined as the presence of air in the pleural cavity. A pneumothorax might occur spontaneously in patients without underlying lung disease (primary spontaneous pneumothorax (PSP)) or in patients with an underlying lung disease (secondary spontaneous pneumothorax), most commonly chronic obstructive pulmonary disease. The underlying cause of spontaneous pneumothorax is likely to be the rupture of "emphysema-like changes" (small bullae or blebs) on the pleural surface, which allows air from the lung into the pleural space. Smoking, male sex and height are the major risk factors for the development of a PSP. There is evidence of a pleural inflammatory reaction in patients with PSP, involving an increase in eosinophils and neutrophils as well as elevated inflammatory cytokines compared with blood levels [1].

From an epidemiological point of view, PSP remains a significant health problem, with an annual incidence of 18-28 per 100000 in males and 1.2-6.0 per 100000 in females [2,3]. Primary therapeutic goals for PSP include removal of air from the pleural space and prevention of recurrences. Air evacuation may be achieved by simple manual needle aspiration, by a small bore tube or a conventional chest tube, the tubes being attached to a one-way valve or to a water-seal device, with or without active suctioning [4].

The treatment of PSP would lend itself well to ambulatory management, since patients are usually young, without relevant comorbidities, and the condition itself caries a low morbidity and mortality [5]. Furthermore, outpatient-orientated treatment rather than hospitalisation might be favoured by many patients and would substantially reduce health-cost expenses. Unfortunately, however, the adherence to recommendations of outpatient management of patients with PSP in clinical practice is rather low $[5,6]$. The lack of clear consensus in treatment probably contributes to both the poor adherence to guidelines and wide variations in practice that are observed worldwide.

In a report published previously in the European Respiratory Journal (ERJ), MARQUETTE et al. [7] showed that a small-calibre catheter attached to a Heimlich valve could be safely proposed as the treatment of choice in hospitalised patients with a large PSP and potentially replace secondary (large-bore) chest tube insertion in those who fail simple manual needle aspiration. The use of a Heimlich valve may offer several advantages when compared with an underwater seal bottle system. It is cheap, less bulky, and enables the patient to be mobile and thus be managed as an outpatient. Despite mixed methodology and a high risk of reporting bias,

Received: Sept 162013 | Accepted: Sept 182013

Conflict of interest: None declared.

Copyright @ERS 2014 
a recent meta-analysis supports the notion that a Heimlich valve might be useful in the ambulatory treatment of PSP, with reasonable treatment success [8].

In this issue of the ERJ, MASSONGO et al. [9] report a prospective study to assess the safety and efficacy of an outpatient treatment algorithm using a small-bore catheter system with a Heimlich valve in selected patients with a PSP. The authors studied 60 consecutive patients who presented to an emergency department of a university hospital with a PSP. Patients with a small PSP had simple clinical observation, whereas those with a large PSP or breathlessness underwent pleural drainage. The aim of the study was to provide outpatient management for both patient groups after a 4-h observation period. In order to achieve this goal, the authors developed "discharge safety criteria". These included the patient's clinical condition, distance from the hospital and/or time of discharge. Patients undergoing pleural drainage for a large pneumothorax, who demonstrated lung re-expansion after $4 \mathrm{~h}$, had their catheter withdrawn and then discharged if these criteria were met. Those who showed incomplete lung re-expansion were discharged with their pleural catheter kept on a Heimlich valve. Importantly, all patients were scheduled for an appointment in the outpatient clinic on the next working day. Patients who were not discharged were kept in hospital for at least one night with further re-evaluation on the next day. The primary end-point of the study was the success rate at day 7 , defined as the percentage of patients with a complete or almost complete persistent lung re-expansion on the chest radiography.

$60 \%$ of the overall patient population (all patients with a small pneumothorax and half of the patients with a "large" pneumothorax) were discharged on the same day. All patients with a small pneumothorax had a persistent complete or almost complete lung re-expansion at the 1-week follow-up without need for further intervention. About $25 \%$ of patients with a large pneumothorax who were discharged initially were readmitted within the first couple of days due to failure of lung re-expansion. Thus, overall, a substantial number of patients were fully managed as outpatients with an estimated cost-saving of more than $€ 100000$ for the population studied according to the authors.

The authors have to be commended for their study as they have provided an important contribution towards implementation of an outpatient management algorithm in a real-life setting. At the same time, their report highlights the challenges one might face in clinical research in this field. First, there is no clear agreement between guidelines from two scientific societies regarding the definition or the first-line treatment of a "large" pneumothorax [5, 10]. While the British Thoracic Society (BTS) recommends manual aspiration first [5], the American College of Chest Physicians suggests a small or large bore chest tube insertion for lung re-expansion [10]. Thus, researchers will always be prone to methodological criticism due to the lack of consensus between these scientific bodies and the absence of a gold standard of treatment with which to compare. Second, the study does not answer the question of whether the single small catheter/Heimlich valve system introduced here should replace manual aspiration completely or whether it should "only" replace large-bore chest tube insertion in those who failed manual aspiration. While the advantage of a "one-system, serial-steps" approach in the report by MASsONGO et al. [9] might appear intuitive, its efficacy and safety have to be formally tested and compared with manual aspiration in an appropriately sized, randomised, controlled, prospective trial. But what would be the primary outcome of such a trial (PSP resolution rate, safety, recurrence rate, patient preference or costs) and who would be willing to finance it? It is very unlikely that randomised controlled trials (RCTs) in this field would be financed by pharmaceutical or medical products companies. At least health care providers should have a genuine interest to cut down costs in the long run and thus be willing to support these kinds of activities. Third, it has to be acknowledged that the management algorithm introduced here might be applicable only to urban areas with similar infrastructure and resources; however, these may differ substantially from one region to another. The outcome of a treatment algorithm might also be influenced by the medical background of the treating physician. PACKHAM and JAISWAL [11], for instance, demonstrated that patients with PSP managed by respiratory physicians had, overall, fewer intercostal drains inserted and a significantly shorter length of hospital stay than those managed by nonrespiratory physicians.

Another set of factors should deserve attention in clinical practice, yet have almost been neglected by the scientific community in the recent past. In this context, the need for oxygen supplementation in the management of patients with a pneumothorax needs to be revisited. If a patient is hospitalised for observation of PSP, supplemental high-flow oxygen is recommended in the BTS guidelines [5]. The evidence related to oxygen therapy beyond correction of arterial hypoxaemia, however, is poor and based on small cohorts $[12,13]$. In the report by MASSONGO et al. [9], oxygen was administered only for correction of arterial hypoxaemia, but not in every patient. Similarly, the effectiveness of smoking cessation on resolution and recurrence rates should be investigated systematically. In a retrospective analysis of 275 patients with PSP, smoking cessation was associated with a reduced risk of recurrence [14]. Despite the apparent relationship between smoking and pneumothorax, $>80 \%$ of young patients continue to smoke 
after their first episode of PSP [15]. Finally, treatment options for PSP based on symptoms only, rather than chest-radiographic appearances, may need to be investigated in future RCTs. After all, it might be argued that a patient with a small pneumothorax may not require radiological or clinical follow-up beyond the first few hours if asymptomatic, yet this hypothesis needs to be determined. Until then, the message delivered in the report by MASSONGO et al. [9] is a good one, but we need to do a better job in the future in order to achieve the best possible care for our patients with PSP.

\section{References}

1 De Smedt A, Vanderlinden E, Demanet C, et al. Characterisation of pleural inflammation occurring after primary spontaneous pneumothorax. Eur Respir J 2004; 23: 896-900.

2 Melton LJ 3rd, Hepper NG, Offord KP. Incidence of spontaneous pneumothorax in Olmsted County, Minnesota: 1950 to 1974. Am Rev Respir Dis 1979; 120: 1379-1382.

3 Gupta D, Hansell A, Nichols T, et al. Epidemiology of pneumothorax in England. Thorax 2000; 55: 666-671.

4 Tschopp JM, Rami-Porta R, Noppen M, et al. Management of spontaneous pneumothorax: state of the art. Eur Respir J 2006; 28: 637-650.

5 MacDuff A, Arnold A, Harvey J, et al. Management of spontaneous pneumothorax: British Thoracic Society Pleural Disease Guideline 2010. Thorax 2010; 65: Suppl. 2, ii18-ii31.

6 Mendis D, El-Shanawany T, Mathur A, et al. Management of spontaneous pneumothorax: are British Thoracic Society guidelines being followed? Postgrad Med J 2002; 87: 80-84

7 Marquette CH, Marx A, Leroy S, et al. Simplified stepwise management of primary spontaneous pneumothorax: a pilot study. Eur Respir J 2006; 27: 470-476.

8 Brims FJ, Maskell NA. Ambulatory treatment in the management of pneumothorax: a systematic review of the literature. Thorax 2013; 68: 664-669.

9 Massongo M, Leroy S, Scherpereel A, et al. Outpatient management of primary spontaneous pneumothorax: a prospective study. Eur Respir J 2014; 43: 582-590.

10 Baumann MH, Strange C, Heffner JE, et al. Management of spontaneous pneumothorax: an American College of Chest Physicians Delphi consensus statement. Chest 2001; 119: 590-602.

11 Packham S, Jaiswal P. Spontaneous pneumothorax: use of aspiration and outcomes of management by respiratory and general physicians. Postgrad Med J 2003; 79: 345-347.

12 Northfield TC. Oxygen therapy for spontaneous pneumothorax. BMJ 1971; 4: 86-88.

13 Chadha TS, Cohn MA. Noninvasive treatment of pneumothorax with oxygen inhalation. Respiration 1983; 44: $147-152$.

14 Sadikot RT, Greene T, Meadows K, et al. Recurrence of primary spontaneous pneumothorax. Thorax 1997; 52: 805-809.

15 Smit HJ, Chatrou M, Postmus PE. The impact of spontaneous pneumothorax, and its treatment, on the smoking behaviour of young adult smokers. Respir Med 1998; 92: 1132-1136. 